\title{
Correlação entre Padrões de Crescimento Facial e Terceiros Molares Inclusos
}

\author{
Correlation between Facial Growth Patterns Andincluded Third Molars
}

\author{
AMANDA DE OLIVEIRA CÂMARA ${ }^{1}$ \\ GREGÓRIO MÁRCIO DE FIGUERÊDO RODRIGUES ${ }^{2}$ \\ KARINA JERÔNIMO RODRIGUES SANTIAGO DE LIMA ${ }^{3}$ \\ RICARDO VILLAR BELTRÃO ${ }^{4}$ \\ REJANE TARGINO SOARES BELTRÃO ${ }^{3}$
}

\section{RESUMO}

Objetivos: Analisar a prevalência dos padrões de crescimento facial e sua correlação com terceiros molares inclusos. Materiais e Métodos: A amostra, composta por 40 pacientes, foi obtida em um centro de radiologia odontológica, a partir deum banco de dados de arquivos de 800 pacientes, obedecendo a critérios de inclusão como: idade acima de 19 anos; presença de telerradiografia em norma lateral e uma radiografia panorâmica, obtidas na mesma época; presença de, no mínimo, um terceiro molar incluso com ápices dentários completamente fechados; permanência de todos os elementos dentários no lado do hemi-arco do elemento incluso; ausência de tratamento ortodôntico prévio. As telerradiografias foram utilizadas para a análise cefalométrica, utilizando-se os ângulos FMA, SN.GoMe e SN.Gn, interpretados de acordo com o padrão USP de análise cefalométrica e comparados com as normas préestabelecidas por essa análise, para a classificação dos padrões faciais em braquicefálico, mesocefálico ou dolicocefálico. A presença e a distribuição dos terceiros molares inclusos foram observadas com a utilização de radiografias panorâmicas. A correlação entre os ângulos dos padrões faciais e os terceiros molares inclusos foi verificada através do teste de Spearman $(p<0,05)$. Dos pacientes pesquisados, $40 \%$ apresentaram perfil dolicocefálico, $35 \%$ mesocefálico e $25 \%$ braquicefálico. Conclusão:Não se constatou nenhuma significância para a correlação dos padrões faciais, nos três ângulos avaliados, com o número de terceiros molares inclusos.

\section{DESCRITORES}

Dente Não Erupcionado. Dente Molar. Ortodontia.

\begin{abstract}
Objective: This study analyzes the prevalence of facial growth patterns and their possible correlation with included third molars. Material and Methods: The sample consisted of 40 patients from a file database of 800 patients, referred to a dental radiology center. The following inclusion criteria were considered: 19 years or older; records including a lateral teleradiograph and a panoramic radiograph obtained at the same time; presence of at least one included third molar with completely closed apexes; presence of all teeth on the hemiarch of the included tooth; no history of orthodontic treatment. Teleradiographs were used for cephalometric analysis considering the angles FMA, SN.GoMe and SN.Gn, interpreted according to the USP standard analysis and compared with pre-established standards for the classification of facial patterns (brachycephalic, mesocephalic or dolichocephalic). The presence and distribution of third molars were observed using panoramic radiographs. The correlations between the angles of the facial patterns and the presence of included third molars was analyzed using Spearman's test $(p<0.05)$. Conclusion: The patients' profiles identified were as follows: $40 \%$ dolichocephalic, $35 \%$ mesocephalic and $25 \%$ brachycephalic. No significant correlation was found between the facial patterns - for all the three angles analyzed - and the number of included third molars.
\end{abstract}

\section{DESCRIPTORS}

Tooth, Unerupted. Molar. Orthodontics.

\footnotetext{
Cirurgiã-dentista João Pessoa. Paraíba Brasil.

Cirurgião-dentista/ João Pessoa. Paraíba. Brasil.

Professora adjunta I da disciplina de Ortodontia da Universidade Federal da Paraíba. João Pessoa. Paraíba. Brasil.

Professor adjunto da disciplina de Radiologia I da Universidade Federal da Paraíba. João Pessoa. Paraíba. Brasil.
} 
$\mathrm{O}$ terceiro molar é o último elemento dentário a erupcionar, surgindo na cavidade bucal por volta dos 18 anos de idade 1 . Por muitas vezes ele encontra-se impedido de erupcionar devido a alguma retenção ou impactação ${ }^{2}$. Dentes retidos são aqueles que, uma vez chegada à época normal em que deveriam irromper, ficam retidos parcialmente, quando ocorre o rompimento da mucosa suprajacente, ou totalmente no interior do osso, com manutenção ou não da integridade do saco pericoronário ${ }^{2}$. Sua frequência é maior na mandíbula do que na maxila, ${ }^{3}$ e menor quanto mais próximo da ausência de um elemento dentário em um hemi-arco onde estiver o terceiro molar, devido à movimentação dentária para o fechamento do espaço ${ }^{2-6}$.

A frequência de impactações de terceiros molares é maior do que em qualquer outro elemento, estando mais associada com a falta de espaço entre a distal do segundo molar e a borda anterior do ramo ascendente ${ }^{3,7-9}$, presente em $90 \%$ dos casos $^{8}$. Portanto, para que ocorra a erupção, é necessário que o diâmetro mesiodistal deste elemento seja inferior ao comprimento retromolar ${ }^{10}$.

Os terceiros molares são indicados para extração quando apresentam infecções, lesões de cárie não restauradas, cistos, tumores ou destruição do osso e dentes adjacentes. Quando se trata de elementos assintomáticos impactados, sua remoção é duvidosa ${ }^{11}$. Cerca de $8 \%$ dos segundos molares superiores e 9,5\% dos inferiores apresentaram sinais de reabsorção provocados por terceiros molares assintomáticos inclusos, além da presença de reabsorção nos ossos da maxila e mandíbula, na região distal a esses elementos. Como não é possível prever o aparecimento de patologias relacionadas à impactação, ele deve ser proservado e o paciente monitorado a cada dois anos após o tratamento ortodôntico, a fim de verificar a condição e a posição de erupção ${ }^{11}$.

Por outro lado, é importante que se possa determinar quais terceiros molares retidos devem ser removidos, pois, quanto mais jovem o paciente for durante a extração, menores complicações irão ocorrer. A previsão de quais terceiros molares permaneceriam retidos e progrediriam para estados de doença, ou desenvolveriam sintomas, seria interessante para que pudessem ser removidos ainda quando os riscos fossem mínimos ${ }^{6}$.

Estudos radiográficos constituem o principal meio de observação para esses casos. Radiografias panorâmicas são utilizadas na rotina do tratamento ortodôntico e no diagnóstico de cirurgias orais ${ }^{6}$, podendo ser bons auxiliares para prever uma possível impactação de terceiros molares ainda na adolescência. Porém, essa análise com panorâmicas só pode ser feita a partir dos 20 anos de idade, pois até essa época a posição desses elementos varia bastante, impedindo um diagnóstico mais precoce de impactação ${ }^{10}$.

Após o advento do cefalostato por Broadbent e Hofrath, em 1931, vários autores se propuseram a estudar sobre as análises cefalométricas, procurando aperfeiçoar as medições para um melhor detalhamento da relação entre os dentes e o complexo craniofacial ${ }^{12-15}$.

Em 1972, Ricketts classificou o padrão facial em três tipos: braquicefálico - padrão de crescimento facial com musculatura forte, arco dentário tendendo a apresentar-se quadrado e contornos faciais curtos podendo indicar má oclusão de Classe II, com mordida profunda e deficiência maxilar vertical;mesocefálico - padrão de crescimento em harmonia (sentido horizontal e vertical) e padrão muscularem harmonia; dolicocefálico - predomínio do crescimento vertical da face em relação ao horizontal, arco dentário apresentando-se em forma de $\mathrm{V}$ e usualmente há associação com palato profundo que, na maioria das vezes, está associado a problemas respiratórios e função muscular hipotônica. Assim, cada padrão pode influenciar de uma maneira diferente no plano de tratamento ortodôntico proposto.

O crescimento mandibular é um fator que contribui para a erupção do terceiro molar $^{4}$ e está diretamente relacionado com o tipo de padrão facial. Tal crescimento pode ser vertical ou sagital, estando o primeiro mais associado com a impactação de terceiros molares ${ }^{8}$.

Alguns estudos citam o crescimento mandibular ${ }^{2,4}$ e a redução do espaço entre a distal do segundo molar e o ramo da mandíbula 3 ,7-9,16-18 como fatores primordiais para a retenção de terceiros molares inferiores, fatores esses que podem ser influenciados pelo tipo de padrão de crescimento facial. Sendo assim, a possível correlação entre esses padrões e a presença de terceiros molares inclusos, embora seja um tema bastante pertinente, ainda é objeto de poucas pesquisas ${ }^{7}$.

Tendo em vista essa possível correlação e, portanto, a possibilidade de prever, ainda em idade jovem, qual paciente poderá ter os terceiros molares erupcionados em função e qual poderá tê-los impactados, o presente estudo avaliou a prevalência 
dos padrões de crescimento facial e sua correlação com a presença de terceiros molares inclusos em pacientes de um centro radiológico da cidade de João Pessoa.

\section{MATERIAL E MÉTODOS}

A presente pesquisa foi aprovada pelo Comitê de Ética em Pesquisa do Hospital Universitário Lauro Wanderley, sob o protocolo $n^{\circ} 495 / 11$, CAAE $\mathrm{n}^{\circ}$ 0215.0.426.126-11.

Em um banco de dados de arquivos de 800 pacientes de uma clínica radiológica em João Pessoa, atendidos em um período de 7 meses, foi obtida uma amostra de 40 pacientes (20 homens e 20 mulheres), obedecendo-se os seguintes critérios de inclusão: idade acima de 19 anos; presença de telerradiografia em norma lateral e uma radiografia panorâmica, obtidas na mesma época; presença de, no mínimo, um terceiro molar incluso com ápices dentários completamente fechados; permanência de todos os elementos dentários no lado do hemi-arco do elemento incluso; ausência de tratamento ortodôntico prévio. Os pacientes que constituíram a amostra foram identificados pelo número gerado no momento do cadastro digital na clínica, garantindo o sigilo. Assim, o acesso obtido às radiografias foi apenas da imagem e sua respectiva numeração.

A marcação dos pontos anatômicos nas telerradiografias para a obtenção das medidas cefalométricas se realizou com a utilização do programa de cefalometria computadorizada Radiomemory® (Belo Horizonte/MG) por um único examinador, em um intervalo de dez dias entre $50 \%$ das análises e as outras $50 \%$, evitando-se, assim, fadiga visual e desgaste físico. A classificação do padrão facial foi obtida utilizando-se os ângulos FMA, SN.GoMe e SN.Gn, interpretados de acordo com o padrão USP ${ }^{24}$ de análise cefalométrica e comparados com as normas pré-estabelecidas por ela. Assim, os pacientes da amostra foram divididos em três grupos, de acordo com o seu padrão facial: 1) Braquicefálico, 2) Mesocefálico e 3) Dolicocefálico. Os ângulos cefalométricos utilizados na pesquisa encontram-se descritos na Tabela 1 e esquematizados na Figura 1.

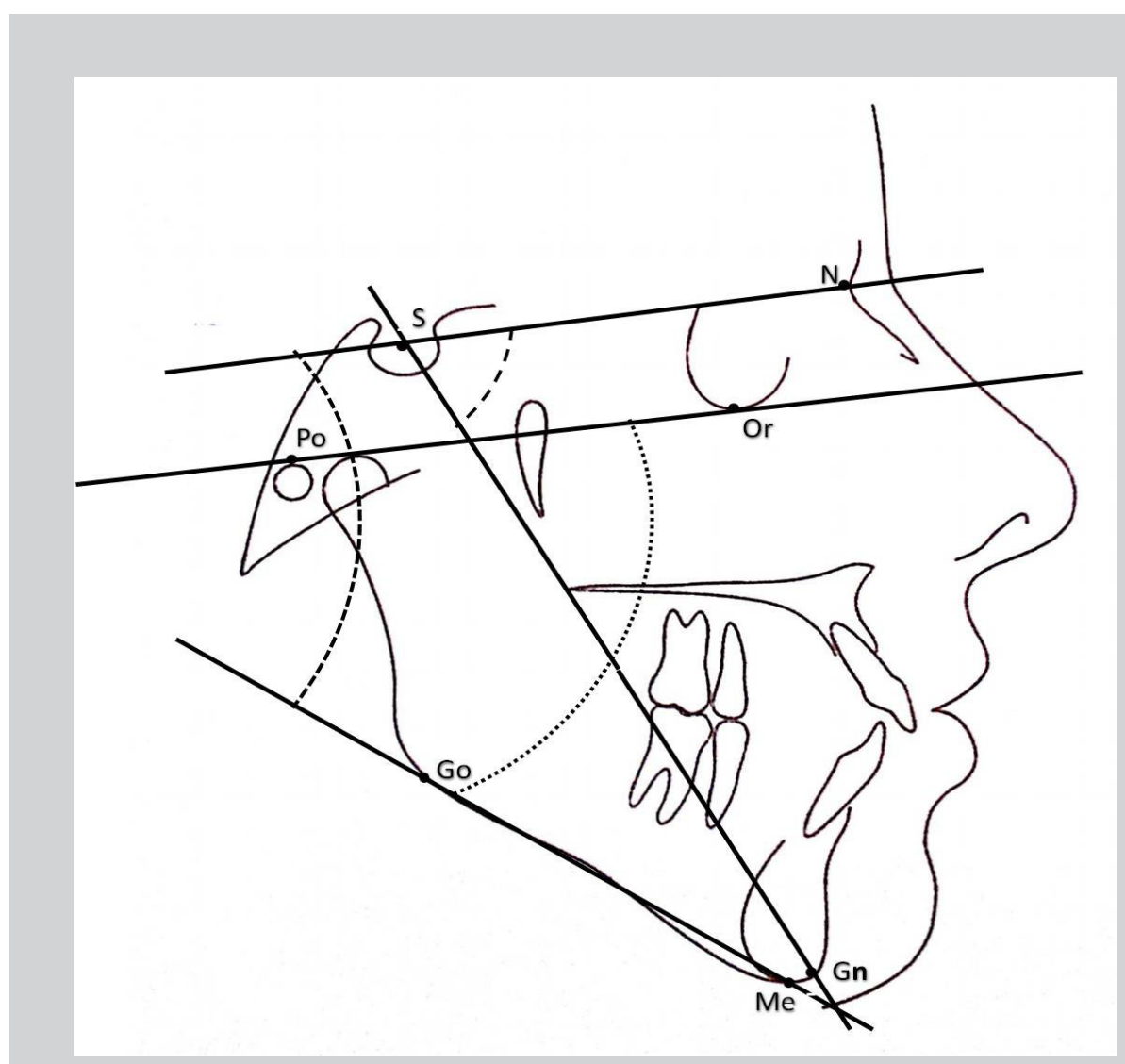

Figura 1: Linhas, planos e ângulos utilizados na análise cefalométrica.

FMA: ângulo formado pelo encontro do plano de Frankfurt com o plano mandibular SN.GoMe: Ângulo formado pelo encontro de $\mathrm{SN}$ e o plano mandibular SN.Gn: Ângulo formado pelo encontro entre SN e SGn 
O erro metodológico para as medidas cefalométricas foi avaliado antes do início do estudo. Para tanto, realizou-se a marcação dos pontos anatômicos e a obtenção das grandezas cefalométricas de 10 pacientes da amostra, escolhidos aleatoriamente. Após um intervalo de 15 dias, as mesmas marcações foram refeitas e novas medidas foram obtidas. Os dados de cada paciente, nas duas datas, foram submetidos ao teste "t" pareado $(p<0,05)$ que demonstrou ausência de significância, garantindo a reprodutibilidade das medidas intra-examinador.

Para avaliar a quantidade e a distribuição de terceiros molares inclusos em cada padrão, tanto na maxila quanto na mandíbula, radiografias panorâmicas digitais, obtidas por meio do softwareRadiocef $®$ (Belo Horizonte/MG) foram utilizadas. Os pacientes de cada grupo foram, então, subdivididos de acordo com o número de terceiros molares inclusos que possuíam, para uma melhor correlação.

A normalidade das medidas cefalométricas foi verificada por meio do teste Shapiro Wilks. Como os dados apresentaram distribuição normal, optouse pela utilização do teste paramétrico de Spearman $(p<0,05)$, correlacionando, assim, o número total de dentes inclusos em cada padrão facial com as três medidas cefalométricas utilizadas.

\section{RESULTADOS}

Em relação à prevalência dos padrões faciais nos pacientes da amostra, $25 \%$ eram braquicefálicos ( $n=10), 35 \%$ mesocefálicos $(n=14)$ e $40 \%$ dolicocefálicos $(n=16)$.

A distribuição dos padrões faciais por gênero está descrita na tabela 1.

A distribuição da frequência de terceiros molares inclusos superiores e inferiores, para cada padrão facial, está representada na tabela 2.

O teste de Spearman revelou não haver correlação estatisticamente significante entre o número de molares inclusos e as três medidas cefalométricas utilizadas (SN.Gn, SN.GoMe e FMA) para os três padrões estudados.

\begin{tabular}{|c|c|c|c|c|c|c|}
\hline \multicolumn{5}{|c|}{ Tabela 1: Distribuição dos padrões faciais por gênero, segundo o } \\
número de indivíduos e a taxa percentual. \\
\hline
\end{tabular}

\begin{tabular}{l|c|c|c|c|c|c|}
\hline \multicolumn{7}{l}{$\begin{array}{l}\text { Tabela 2: Número de molares inclusos maxilares e mandibulares, para cada } \\
\text { padrão facial. }\end{array}$} \\
\hline & Braquicefálico & \multicolumn{2}{c|}{ Mesocefálico } & \multicolumn{2}{c|}{ Dolicocefálico } \\
\hline & $\mathbf{n}$ & $\%$ & $\mathbf{n}$ & $\%$ & $\mathbf{n}$ & $\%$ \\
\hline Maxila & 5 & $12,5 \%$ & 11 & $27,5 \%$ & 19 & $47,5 \%$ \\
\hline Mandíbula & 14 & $35 \%$ & 13 & $32,5 \%$ & 18 & $45 \%$ \\
\hline TOTAL & 19 & $47,5 \%$ & 24 & $60 \%$ & 37 & $92,5 \%$ \\
\hline
\end{tabular}




\section{DISCUSSÃO}

Os terceiros molares mandibulares são um enigma na Ortodontia, pois seu desenvolvimento e seu período de erupção são bastante variáveis ${ }^{4}$. A falta de espaço é considerada por muitos autores o fator primordial para a não erupção desses elementos. Muitos estudos associaram a impactação de terceiros molares mandibulares ao curto espaço alveolar existente entre o segundo molar e o ramo ascendente ${ }^{3,7-9,18}$, e compararam a impactação com a presença de algumas variáveis do crescimento mandibular, sem, contudo, correlacionar diretamente com os padrões de crescimento facial.

Direção de crescimento vertical do côndilo², $8,18,19$, aumento da largura anteroposterior do ramo mandibular devido a uma falha no processo de reabsorção ${ }^{2-4,17}$, maior comprimento do ramo, menor comprimento do $\operatorname{corpo}^{18,20}$ e maior ângulo de crescimento mandibular ${ }^{2}$ são características reportadas em vários estudos como presentes na maioria dos casos de impactação de terceiros molares. Embora essas pesquisas não tenham associado diretamente o tipo de padrão de crescimento facial, é possível observar que, através das variáveis relatadas, o padrão dolicocefálico esteja mais presente nesses casos.

Outros fatores também podem estar associados, como a velocidade de maturação dos terceiros molares, a inclinação mesial desses elementos e o tamanho da dentição em relação aos ossos maxilare ${ }^{4,8,9,18,20}$, apontando que fatores dentários e esqueléticos diversos estão envolvidos na sua impactação. Vários tipos de amostras e diferentes características foram analisadas, não havendo uma padronização entre os estudos , $^{4,9,9,18,20}$.

A associação direta entre os tipos de padrões faciais propostos por Rickettse a ausência de erupção dos terceiros molares foi motivo de estudo ${ }^{7}$, concluindo que, devido ao maior potencial de crescimento mandibular e uma maior reabsorção da borda anterior do ramo, pacientes braquifaciais possuem um espaço adicional para a erupção dos terceiros molares. Assim, os dolicocefálicos, que não possuem tais características, apresentaram o maior índice de impactações ${ }^{21}$.

Na presente pesquisa, embora tenha existido uma tendência maior de impactação de terceiros molares para o padrão dolicocefálico, não houve correlação estatística entre os padrões e a ausência da erupção desses elementos, discordando dos estudos que associaram as variáveis do padrão dolicocefálico de crescimento com a presença de impactação ${ }^{2-4,7,8,17-20}$. Tal discordância entre os resultados pode ser justificada pela diferença étnica entre as populações estudadas ${ }^{2,3}$ ou pelo maior número de amostras utilizadas nas outras pesquisas ${ }^{3,4,17,18}$, sugerindo que um aumento no número das amostras poderia confirmar essa tendência e concordar com tais estudos.

Assim como em outras pesquisas ${ }^{7,10}$, não se encontrou predileção por gênero, tendo homens e mulheres as mesmas chances de apresentarem terceiros molares impactados. Já em relação à maxila e à mandíbula, os terceiros molares inferiores apresentaram maior frequência de impactação do que os superiores, concordando com outro resultado encontrado na literatura ${ }^{3}$, o que pode ser atribuído às diferenças na composição óssea e às inúmeras variáveis do crescimento mandibular.

Em outras pesquisas também não foi encontrado um padrão de crescimento mandibular específico nos casos de impactação de terceiros molares ${ }^{11}$, logo, não há um padrão facial associado para esses casos. O tamanho do espaço retromolar não interferiu nos casos de impactação ${ }^{10}$ e o comprimento mandibular não foi menor nos indivíduos que apresentavam retenção desses elementos ${ }^{23}$.

A amostra da presente pesquisa foi constituída por pacientes que, dentre os critérios de inclusão, não apresentavam ausências ou extrações de elementos dentários no mesmo hemiarco que o terceiro molar impactado e nem haviam se submetido a tratamento ortodôntico prévio. Isso porquequanto mais próximo de uma ausência dentária, maiores são as chances de erupção ${ }^{2-6}$, e que o movimento mesial dos molares durante o tratamento previne a impactação, reduzindo seu risco de $22 \%$ a $34 \%$ para cada milímetro ganho durante a movimentação ${ }^{17}$. Portanto, é importante que se conheça o padrão de erupção, o desenvolvimento e o prognóstico dos terceiros molares para que um tratamento mais adequado, com ou sem extrações e mesializações, seja proposto.

Apesar de não se ter estabelecido uma correlação significante com o padrão dolicocefálico, observou-se uma tendência das impactações dos terceiros molares com esse tipo de padrão facial. Sendo assim, pesquisas futuras são necessárias para que se reforce a real relação entre os padrões de crescimento facial e a presença de terceiros molares impactados, auxiliando no diagnóstico precoce e no prognóstico do tratamento ortodôntico. 


\section{CONCLUSÃO}

Embora tenha existido uma tendência maior de impactação de terceiros molares para o padrão dolicocefálico, não se constatou nenhuma significância estatística para a correlação dos padrões faciais, nos três ângulos avaliados.

\section{REFERÊNCIAS}

1. Chung $\mathrm{CJ}$, Han JH, Kim KH. The pattern and prevalence of hypodontia in Koreans. Oral diseases. 2008; 14(7):620-25.

2. Kaplan RG. Some factors related to mandibular third molar impaction. Angle Orthodontic. 1975;45(3):153-58.

3. Hassan AH. Mandibular cephalometric characteristics of a Saudi sample of patients having impacted third molars. The Saudi Dental Journal. 2010;23(2):73-80.

4. Richardson ME. Some aspects of lower third molar erupcin. Angle Othodontist. 1974;42(4):368-86.

5. Kim TW, Artun J, Behbehani F, Artese F. Prevalence of third molar impaction in orthodontic patients treated nonextraction and with extraction of 4 premolars. Am J Orthod Dentofacial Orthop. 2003;123(2):138-45.

6. Ventã I. How Often do asymptomatic, disease-free third molars need to be removed? J Oral Maxillofac Surg. 2012;70(Suppl 1):41-47.

7. Breik $O$, Grubor D. The incidence of mandibular third molar impactions in different skeletal face types. Australian Dental Journal. 2008;53(4):320-24.

8. Björk A, Nensen E, Palling M. Mandibular grows and third molar impactions. Acta odontologica scandinávica. 1956;14(3):231-72.

9. Ricketts RM. A principle of arcial growth of the mandible. Angle Orthodontist. 1972;42(4):141-45.

10. Legovic' M, Legovic' I, Brumini G, Vancjura I, C'abov T, Ovesnik $\mathrm{M}$, et al. Correlation between the pattern of facial growth and the position of the mandibular third molar. Journal of Oral Maxillofacial Surgeons. 2008;66(6):1218-24.

11. Kahl KL, Gerlach RD, Hilgers D. Along-term, follow-up radiographic evaluation of asymptomatic impacted third molars in orthodontically treated patients. International journal of Oral and Maxilofacial Sugery. 1994;23(5):27985.

12. Downs WB. The role of cephalometrics in orthodontic case analysis and diagnosis. . American Journal of Orthodonics. 38(3):162-82.

13. Twed $\mathrm{CH}$. The Frenkfurt-mandibular Incisor Angle (FMIA) In the Orthodontic Diagnosis, Treatment Planning and Prognosis. Angle Orthodontist. 1954;24(3):121-69.

14. McNamara Jr JA. Amethod of cephalometric evaluation. American Journal of Orthodonics. 1984;86(6):449-69.
15. Steiner CC. Cephalometric for you and me. American Journal Orthodontic and Dentalfacial Orthopedics. 1953;38(10):729-55.

16. Ledyard BC. A study of the mandibularthirdmolararea. American Journal of orthodontics. 1953;39(4):366-73.

17. Artun J, Behbehani F, Thalib L. Prediction of maxillary third molar impaction in adolescent orthodontic patients. Angle Othodontist. 2005;75(6):47-55.

18. Capelli JJ. Mandibular growth and third molar impaction in extraction cases. Angle Othodontist. 1991;61(3):22329.

19. Björk A, Skiller V. Facial development and tooth eruption: an implant study at the age of puberty. Am J Orthod Dentofacial Orthop. 1972;62(4):339-83.

20. Björk A. Variations in the growth pattern of the human mandible: longitudinal radiographic study by the implant method. J Dent Res. 1963;42(1):400-11.

21. Nanda SK. Patterns of vertical growth in the face. Am J Orthod Dentofacial Orthop. 1988;93(2):103-16.

22. Ades AG. A long-term study of the relationship of third molars to changes in the mandibular dental arch. American Journal of Orthodonics and Dentofacial Orthopedics. 1990;97(4):323-35.

23. Dierkes D. An investigation of the mandibular third molars in orthodontic cases. Angle Orthod. 1975;45(3):20712.

24. Interlandi S, Sato-tsuji A. Projeção USP na relação cefalométrica maxilo-mandibular. Ortodontia: bases para a iniciação. 4. ed. São Paulo: Artes Médicas, 1999;22537.

\section{Correspondência}

Amanda de Oliveira Câmara

Endereço: Rua Maria Caetano Fernandes de Lima, 396, Tambauzinho

CEP: 58042-050.

João Pessoa. Paraíba. Brasil.

E-mail: amanda_camara@hotmail.com 